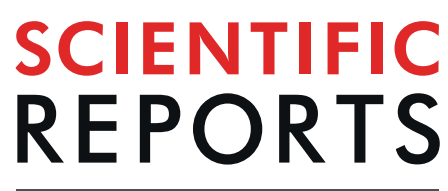

natureresearch

\title{
Cerebrospinal fluid proteomics implicates the granin family in Parkinson's disease
}

\author{
Melissa S. Rotunno ${ }^{1,2}$, Monica Lane ${ }^{2}$, Wenfei Zhang ${ }^{3}$, Pavlina Wolf ${ }^{2,8}$, Petra Oliva ${ }^{2,9}$, \\ Catherine Viel ${ }^{1}$, Anne-Marie Wills ${ }^{6}$, Roy N. Alcalay $\mathbb{D}^{5}$, Clemens R. Scherzer $\mathbb{D}^{4,6,7}$, \\ Lamya S. Shihabuddin ${ }^{1}$, Kate Zhang ${ }^{2,8}$ \& S. Pablo Sardi $\mathbb{1}^{1 *}$
}

Parkinson's disease, the most common age-related movement disorder, is a progressive neurodegenerative disease with unclear etiology. Better understanding of the underlying disease mechanism(s) is an urgent need for the development of disease-modifying therapeutics. Limited studies have been performed in large patient cohorts to identify protein alterations in cerebrospinal fluid (CSF), a proximal site to pathology. We set out to identify disease-relevant protein changes in CSF to gain insights into the etiology of Parkinson's disease and potentially assist in disease biomarker identification. In this study, we used liquid chromatography-tandem mass spectrometry in dataindependent acquisition (DIA) mode to identify Parkinson's-relevant biomarkers in cerebrospinal fluid. We quantified 341 protein groups in two independent cohorts $(n=196)$ and a longitudinal cohort ( $n=105$ samples, representing 40 patients) consisting of Parkinson's disease and healthy control samples from three different sources. A first cohort of 53 Parkinson's disease and 72 control samples was analyzed, identifying 53 proteins with significant changes $(p<0.05)$ in Parkinson's disease relative to healthy control. We established a biomarker signature and multiple protein ratios that differentiate Parkinson's disease from healthy controls and validated these results in an independent cohort. The second cohort included 28 Parkinson's disease and 43 control samples. Independent analysis of these samples identified 41 proteins with significant changes. Evaluation of the overlapping changes between the two cohorts identified 13 proteins with consistent and significant changes $(p<0.05)$. Importantly, we found the extended granin family proteins as reduced in disease, suggesting a potential common mechanism for the biological reduction in monoamine neurotransmission in Parkinson's patients. Our study identifies several novel protein changes in Parkinson's disease cerebrospinal fluid that may be exploited for understanding etiology of disease and for biomarker development.

Parkinson's disease (PD) is the second most frequent neurodegenerative disease affecting approximately 1 million people in the US. Although the etiology of PD is largely unknown, genetic and environmental factors have been implicated in disease development. The pathological hallmarks of PD are dopaminergic neuronal loss in the substantia nigra pars compacta and presence of Lewy bodies containing aggregated $\alpha$-synuclein ${ }^{1-3}$. While there are medications to improve motor function in PD, there are no FDA approved interventions to slow down or reverse the disease because of our limited understanding of disease pathogenesis ${ }^{2-4}$. Identifying protein alterations present in PD could provide insight into the underlying mechanism of disease and aid in the development of future novel therapeutics.

Another major challenge of disease modification trials is the lack of objective measurements of disease progression. Current biomarkers are clinical assessments, which are of limited use because of high variability in

${ }^{1}$ Rare and Neurologic Diseases Therapeutic Area, Sanofi, Inc., Framingham, MA, 01701, USA. ${ }^{2}$ Biomarkers and Bioanalytics, Translational Sciences, Sanofi, Inc., Framingham, MA, 01701, USA. ${ }^{3}$ Translational Medicine, Sanofi, Inc., Framingham, MA, 01701, USA. "Precision Neurology Program, Harvard Medical School, Brigham \& Women's Hospital, Boston, MA, 02115, USA. ${ }^{5}$ Department of Neurology, Columbia University, New York, NY, 10032-3784, USA. ${ }^{6}$ Department of Neurology, Massachusetts General Hospital, Boston, MA, 02114, USA. ${ }^{7}$ APDA Center for Advance Parkinson Research, Harvard Medical School, Brigham \& Women's Hospital, Boston, MA, 02115, USA. ${ }^{8}$ Present address: Editas Medicine, Cambridge, MA, 02141, USA. ${ }^{9}$ Present address: ARCHIMED Life Sciences GmbH, Leberstraße 20/2, 1110, Vienna, Austria. *email: pablo.sardi@sanofi.com 


\begin{tabular}{|c|c|c|c|c|c|c|}
\hline \multirow[b]{2}{*}{$\begin{array}{l}\text { Group } \\
\text { (Sample \#) }\end{array}$} & \multicolumn{2}{|l|}{ Cohort 1} & \multicolumn{2}{|l|}{ Cohort 2} & \multicolumn{2}{|c|}{ Longitudinal cohort } \\
\hline & $\begin{array}{l}\mathrm{HC} \\
(\mathrm{n}=72)\end{array}$ & $\begin{array}{l}\mathrm{PD} \\
(\mathrm{n}=53)\end{array}$ & $\begin{array}{l}\mathrm{HC} \\
(\mathrm{n}=43)\end{array}$ & $\begin{array}{l}\mathrm{PD} \\
(\mathrm{n}=28)\end{array}$ & $\begin{array}{l}\mathrm{HC} \\
(\mathrm{n}=60)\end{array}$ & $\begin{array}{l}\mathrm{PD} \\
(\mathrm{n}=45)\end{array}$ \\
\hline Gender $(\mathbf{m} / \mathbf{f})$ & $49 / 23$ & $40 / 13$ & $27 / 16$ & $19 / 9$ & $44 / 16$ & $27 / 18$ \\
\hline $\begin{array}{l}\text { Age } \\
(\text { mean } \pm \mathrm{SD} \text {, range) }\end{array}$ & \begin{tabular}{|l|}
$68 \pm 9$ \\
$(45-83)$ \\
\end{tabular} & $\begin{array}{l}67 \pm 9 \\
(43-84) \\
\end{array}$ & \begin{tabular}{|l|}
$58 \pm 10$ \\
$(36-81)$ \\
\end{tabular} & \begin{tabular}{|l|}
$64 \pm 6$ \\
$(41-72)$ \\
\end{tabular} & \begin{tabular}{|l|}
$56.6 \pm 10.4$ \\
$(35-81)$
\end{tabular} & $\begin{array}{l}63.2 \pm 8.8 \\
(41-75) \\
\end{array}$ \\
\hline $\begin{array}{l}\text { Disease Duration } \\
(\text { mean } \pm \mathrm{SD} \text {, range) }\end{array}$ & - & $\begin{array}{l}5.5 \pm 4.5 \\
(0-20)\end{array}$ & - & $\begin{array}{l}4.3 \pm 3.3 \\
(0-11) \\
\end{array}$ & - & $\begin{array}{l}3.8 \pm 2.4 \\
(0-9)\end{array}$ \\
\hline $\begin{array}{l}\text { HY score } \\
(\text { mean } \pm S D \text {, range })\end{array}$ & - & $\begin{array}{l}2.2 \pm 0.5 \\
(1-4)^{*}\end{array}$ & - & $\begin{array}{l}2.1 \pm 0.6 \\
(1-4)\end{array}$ & - & $\begin{array}{l}2.2 \pm 0.4 \\
(2-3)\end{array}$ \\
\hline $\begin{array}{l}\text { MMSE }^{\mathrm{b}} \\
(\mathrm{mean} \pm \mathrm{SD}, \text { range })\end{array}$ & $\begin{array}{l}29.1 \pm 1.2 \\
(26-30) \\
\end{array}$ & $\begin{array}{l}27.4 \pm 4.0 \\
(14-30)\end{array}$ & $\begin{array}{l}27.8 \pm 1.4^{\mathrm{c}} \\
(25-30)\end{array}$ & $\begin{array}{l}28.0 \pm 2.1^{\mathrm{c}} \\
(23-30) \\
\end{array}$ & $\begin{array}{l}28.7 \pm 1.3 \\
(25-30)\end{array}$ & $\begin{array}{l}29.0 \pm 1.2 \\
(26-30)\end{array}$ \\
\hline $\begin{array}{l}\text { UPDRS total }{ }^{\mathrm{d}} \\
(\text { mean } \pm \mathrm{SD} \text {, range) }\end{array}$ & - & $\begin{array}{l}37.6 \pm 14.8 \\
(15-73) * * \\
\end{array}$ & - & \begin{tabular}{|l|}
$41.2 \pm 16.5$ \\
$(19-98)$
\end{tabular} & - & $\begin{array}{l}40.5 \pm 14.0 \\
(15-73)^{* * *}\end{array}$ \\
\hline \# of Individual Patients & 72 & 53 & 43 & 28 & 22 & 18 \\
\hline$\%$ l-dopa or agonist & - & $91 \%$ & - & $96 \%$ & - & $94 \%$ \\
\hline Source $e^{e}$ & $\begin{array}{l}\mathrm{HBS}=53 \\
\mathrm{PM}=19\end{array}$ & $\begin{array}{l}\mathrm{HBS}=35 \\
\mathrm{PM}=18\end{array}$ & $\begin{array}{l}\mathrm{HBS}=26 \\
\mathrm{CU}=17\end{array}$ & $\begin{array}{l}\mathrm{HBS}=8 \\
\mathrm{CU}=20\end{array}$ & $\mathrm{HBS}=60$ & $\mathrm{HBS}=45$ \\
\hline
\end{tabular}

Table 1. Patient demographics and clinical characteristics of all cohorts. *Data not available for five patient samples in this cohort. **Data not available for nineteen patient samples in this cohort. ***Data not available for one time point for one patient sample. ${ }^{a} \mathrm{HY}$ score, Hoehn and Yahr scale. ${ }^{b} \mathrm{MMSE}$, mini-mental state examination. 'The samples from Columbia University underwent the Montreal Cognitive Assessment (MoCA), not MMSE. ${ }^{\mathrm{d} U P D R S}$ total, unified Parkinson's disease rating scale. ${ }^{e}$ Harvard biomarkers study, HBS; Columbia University, CU; PrecisionMed, Inc., PM.

disease progression, the effect of symptomatic medications on the exam and subjective clinical scoring. The present study set out to identify biomolecular signatures in cerebrospinal fluid (CSF), which could also assist the analysis of therapeutic efficacy and disease modifications in proof of concept trials. The potential utility of CSF biomarkers for neurodegenerative diseases was previously demonstrated by the development of various assays that are currently used for Alzheimer's disease (e.g. total A $\beta 42$ and phosphorylated tau ${ }^{5,6}$.

Quantitative proteomics has made significant progress with the development of liquid chromatography-tandem mass spectrometry (LC/MS/MS) in data-independent acquisition mode (DIA-MS) ${ }^{7}$. DIA-MS provides a suitable compromise of sensitivity, reproducibility, and extent of peptide identification, and is mainly limited by the breadth and quality of the protein library that is required in the data analysis pipeline. While traditional proteomic approaches lack reproducible peptide fragmentation and quantification of lower abundant proteins, DIA-MS allows the quantification of the same set of proteins measured in all samples as long as it is above the lower limit of detection and present in the peptide spectral library used in the data analysis pipeline. We selected the PD-relevant biofluid CSF for identifying novel protein changes. Analysis of the CSF proteome presents a unique set of challenges to overcome when interpreting the data output. Simple normalization of protein quantification to CSF volume, for example, adds analytical error, as the total protein concentration varies 3 -fold between subjects, ranging from 0.15 to $0.5 \mathrm{mg} / \mathrm{ml}^{8}$. Additionally, total protein normalization mainly reflects the concentration of the most abundant proteins, albumin and cystatin-C, both of which are reportedly altered in $\mathrm{PD}^{9-12}$. DIA-MS permits the quantification of hundreds of proteins in a single run, allowing for a normalization approach that considers the intensities of all detectable proteins with a more evenly weighted distribution ${ }^{13}$. Considering these advantages and disadvantages, we initially focused on DIA-MS to obtain the best compromise between sensitivity, reproducibility, and extent of peptide identification for quantifying the CSF proteome.

In the present study, we set out to identify protein alterations in PD by utilizing DIA-MS to analyze CSF samples in two independent cohorts consisting of PD and healthy control (HC) subjects. We developed a CSF processing pipeline with reproducible protein quantification and minimal CSF input. Several novel protein aberrations were identified. The changes implicate altered production/processing of biologically active peptides in the extended granin family, cell adhesion, and insulin regulation. Additionally, by employing univariate analyses, LASSO regression and multivariate analyses, we identified a unique biomarker signature and protein ratios that separate PD from $\mathrm{HC}$ in both cohorts.

\section{Methods}

Subjects and sample collection. We analyzed samples collected from 196 participants including 115 HC and $81 \mathrm{PD}$ recruited from three separate sources (Table 1). All subjects provided their gender, age, date of birth, and ethnic background. Patients underwent cognitive function assessment (MMSE: mini-mental state examination; or MoCA: Montreal Cognitive Assessment) and evaluation of disease progression by both the unified Parkinson's disease rating scale (UPDRS) and Hoehn and Yahr (HY) scoring, except where noted. Principal component analysis did not reveal any batch effect based on CSF source (Additional File 1: Fig. S1).

CSF donors were originally enrolled in the following studies:

(1) Harvard Biomarkers Study (HBS) $)^{14-16}: 79 \mathrm{HC}$ and 43 PD were included. A subset of patients, referred to as the longitudinal cohort, $(\mathrm{HC}=22$ subjects, representing 60 samples, and $\mathrm{PD}=18$ patients, representing 45 samples) donated 2-4 CSF samples with intervals of approximately one year between each collection (See Table 1). 
(2) Columbia University: $17 \mathrm{HC}$ and $20 \mathrm{PD}$ who participated in the Biofind ${ }^{17}$ study at Columbia University were included. Participants were examined and donated spinal fluid in the "off" state, defined as withholding overnight dopaminergic medications.

(3) PrecisionMed, Inc.: $19 \mathrm{HC}$ and 18 PD CSF samples were included. Inclusion criteria for the study required at least a $1 \mathrm{yr}$ PD diagnosis with stable response to dopamine therapy for $>6 \mathrm{mo}$.

Sample collection for biochemical analysis was respectively approved by Partners HealthCare IRB, Columbia University IRB, and PrecisionMed IRBs, and all participants signed an informed consent. All methods were compliant with Sanofi's guidelines and regulations.

In all cases, CSF was obtained through lumbar puncture and pooled at room temperature. Collected CSF was centrifuged at $400-2000 \mathrm{~g}$ for $10 \mathrm{~min}$ and stored at $-80^{\circ} \mathrm{C}$. The time interval between collection and storage varied between CSF source (HBS: $<2 \mathrm{~h}$; CU: $<15 \mathrm{~min}$; PM: $<1 \mathrm{~h}$ ).

CSF processing. The CSF samples were thawed on ice and aliquoted prior to use. CSF protein concentrations were determined with a Micro BCA Protein assay kit (Pierce). Approximately $20 \mu \mathrm{g}$ of total protein containing Halt ${ }^{\mathrm{TM}}$ protease and phosphatase inhibitor (ThermoFisher) was subjected to acetone precipitation. The sample was reduced with dithiothreitol, alkylated with iodacetamide and digested with recombinant LysC (1:50, Promega) and trypsin (1:25, Roche Diagnostics) in $0.1 \%$ Rapigest (Waters) in $50 \mathrm{mM}$ Ammonium bicarbonate. Rapigest was precipitated with $2 \%$ formic acid and removed by centrifugation. Samples were dried and resuspended in $40 \mu \mathrm{l}$ of $3 \%$ acetonitrile and $0.1 \%$ formic acid.

LC/MS/MS analysis. Liquid chromatography-tandem mass spectrometry (LC/MS/MS) was performed on a Q Exactive HF hybrid Quadrupole-Orbitrap mass spectrometer (ThermoFisher) interfaced with NanoAcquity (Waters). The sample was separated using a C18 trapping (2GVM Trap Symmetry C18 column, $180 \mu \mathrm{m} \times 20 \mathrm{~mm}$, Waters) and reverse phase column ( $1.8 \mu \mathrm{m}$ HSS T3 nanoACQUITY column, $100 \mu \mathrm{m} \times 100 \mathrm{~mm}$, Waters) for DDA and DIA acquisition over a $60 \mathrm{~min}$. gradient. MS/MS spectra were acquired with top 20 ions for DDA mode with MS1 resolution of 120,000 (automatic gain control $(\mathrm{AGC})=3 \mathrm{e} 6$ ) and MS2 resolution of 15,000 (AGC=1e5). An inclusion list containing 17 precursors ranging from $\mathrm{m} / z 400$ to 1000 with varying isolation windows was used for DIA with a resolution of $60,000(\mathrm{AGC}=3 \mathrm{e} 6)$.

DIA-MS data processing for library generation. Raw DDA files were processed in Proteome discoverer 1.4 (Thermo). Peptide identification was performed using Mascot v2.4 (Matrix Science Ltd) search against the UniProt human database (www.uniprot.org) with peptide mass tolerance of $10 \mathrm{ppm}$ and fragment ion tolerance of $20 \mathrm{mmu}$. Carbamidomethyl (C) was included as a fixed modification and oxidation (M), deamidation $(\mathrm{N}, \mathrm{Q})$, phosphorylation $(\mathrm{S}, \mathrm{T})$, glutamine to pyroglutamate $(\mathrm{N}$-term), acetyl $(\mathrm{N}$-term), and oxidation $(\mathrm{H}, \mathrm{W})$ were included as variable modifications. The output file was imported into Spectronaut to generate the library with a maximum missed cleavage of 2, peptide length of 6 to 47 amino acid residues.

DIA-MS data processing for CSF sample analysis. Samples were processed in Spectronaut Pulsar v11 utilizing the aforementioned library. Peptide precursor identification was set with q-value cutoff of 0.01 , corresponding to a false discovery rate (FDR) of $1 \%$. Endogenous peptides were used for retention time calibration across samples. Intensities of the top 3 peptide precursors identified for each protein were averaged, when available, to generate the protein level quantification. A local normalization approach was employed that incorporates local regression with locally weighted smoothing as previously described ${ }^{13}$. The variability of the distribution of protein intensities amongst samples is reduced following normalization (Additional File 1: Fig. S2). A QC standard that was processed repeatedly on multiple days and with different batches of CSF demonstrates that 191 proteins have a CV $<20 \%$ for protein quantification (Additional File 1: Fig. S3), with an overall median CV of $18 \%$.

Statistical analysis of cohorts for differentiating PD from HC. For univariate analysis to identify p-value, odds ratio, and AUC (area under the receiver operating characteristic (ROC) curve), a logistic regression model was fitted for each individual protein. False discovery rate ${ }^{18}$ is applied to adjust the multiplicity. The response variable was the binary indicator of PD status. Model covariates consisted of individual proteins. The cohorts were analyzed separately. For peptide quantification of granins, a two-tailed t-test was preformed assuming unequal variance.

Statistical analysis of cohorts for biomarker signature development. Using Cohort 1 as the training dataset, 5 proteins identified by LASSO (least absolute shrinkage and selection operator) regression were used to build a predictive model using a multivariate logistic model to develop a biomarker signature. LASSO is a penalized regression method for selecting import variables and providing importance index for the variables ${ }^{19}$. Only proteins that were quantifiable in at least $90 \%$ of samples were included in the analysis (Additional File 2 and 3). The predictive model from Cohort 1 was applied to Cohort 2 for evaluation. R 3.4 software was used for the statistical analysis.

For detailed methods see Additional File 4.

Ethics approval and consent to participate. Sample collection for biochemical analysis was approved by IRBs at Partners HealthCare, Columbia, and PrecisionMed (Protocols 7800 and 1009). All participants signed informed consents. 


\section{Results}

Quantitative proteomics reveals unique biomarkers in PD. To identify novel PD-relevant protein changes in CSF and unique biomarker signatures, we employed a DIA-MS workflow (Fig. 1a). DIA-MS requires a peptide ion library to process and interpret complex spectra acquired. To develop a comprehensive peptide library, a subset of CSF samples were processed and analyzed by LC/MS/MS in DDA mode (see methods, Fig. 1b,c). The resulting library consisted of 2632 unique peptides, representing 341 quantifiable protein groups (Fig. 1d) ${ }^{20,21}$. Of note, several proteins representing PD-related pathways were included in the protein library, such as neuroinflammation and mitochondrial dysfunction (Fig. 1d, right). The limitations of the mass spectrometry approach employed in this study precluded the detection of several known PD-relevant proteins such as $\alpha$-synuclein, tau, and neurofilament which have been extensively quantified in multiple cohorts ${ }^{22,23}$. As expected with the low volumes of CSF used in this analysis (15-50 $\mu \mathrm{l} / \mathrm{sample})$, known exosome associated proteins, such as flotillin-1 (FLOT1) and tumor susceptibility gene (TSG101), were not detected. The absence of these proteins suggests that the exosomal proteome has minimal contribution to the protein quantification described in this study $^{24}$. An initial "discovery" cohort (Cohort 1) of 72 HC and 53 PD samples was analyzed by DIA-MS (Fig. 1e, Table 1). Quantification of 341 protein groups in the discovery set identified 53 proteins that were differentiated between PD and HC with a p $<0.05$ by logistic regression (Additional File 2). A second "test" cohort (Cohort 2) consisting of $43 \mathrm{HC}$ and $28 \mathrm{PD}$ identified 41 proteins with $\mathrm{p}<0.05$ (Additional File 3 ). Univariate analysis and logistic regression of all 341 protein groups identified 14 proteins with an absolute odds ratio $>2$ or $<0.5$, and AUC $>0.6$ in both cohorts (Additional File 1: Fig. S4, Additional File 5). The best performing single protein using these criteria was apolipoprotein D (APOD) with an AUC of 0.68 and 0.69 in the ROC analysis for Cohort 1 and 2, respectively (Additional File 1: Fig. S4). Interestingly, APOD has also been shown to be increased in plasma of $\mathrm{PD}$ patients ${ }^{25}$.

While APOD demonstrated relatively high specificity and sensitivity for a single analyte, alternative differences in disease and control groups could account for this, requiring a more robust normalization. To eliminate this potential confounding factor, we explored the predictive value of protein ratios. Using Cohort 1 as a discovery cohort, APOD and attractin (ATRN) were identified as the top 2 increasing proteins and secretogranin-2 (SCG2) and cadherin-2 (CDH2) as the top 2 decreasing proteins based on an AUC $>0.65$ and the best odds ratio from the univariate analysis (Fig. 2a,b). The protein ratios of ATRN/SCG2 and APOD/CDH2 were obtained with the improved AUC of 0.78 and 0.71 in Cohort 1, respectively. However, the ATRN/SCG2 protein ratio had limited predictive value in Cohort $2(\mathrm{AUC}=0.61)$, demonstrating a lack of consistency between $\mathrm{PD}$ populations. The second protein ratio, APOD/CDH2 maintained high predictive values for Cohort 2 with an AUC of 0.72, demonstrating a better predictive value over APOD alone (Fig. 2c,d).

To identify a more advanced biomarker signature that incorporates multiple proteins for increased predictive value, we performed LASSO regression and multivariate analysis on Cohort 1. A biomarker signature consisting of 5 proteins (APOD, SCG2, complement C1q subcomponent subunit C (C1QC), ATRN, and fibulin-1 (FBLN1)) was identified that separated PD from HC with an AUC of 0.84 (Fig. 2e). When the biomarker signature was applied to Cohort 2, ROC analysis determined an AUC of 0.67. The reduced predictive value in the test cohort may be due, in part, to the heterogeneity of the PD population. When we apply the biomarker signature to a longitudinal cohort consisting of $22 \mathrm{HC}$ and $18 \mathrm{PD}$ with repeat yearly visits, consistent elevation in PD compared to $\mathrm{HC}$ is observed (Fig. 2f). Larger longitudinal analyses of fast and slow progressing PD populations are required to establish whether these could be effective biomarker candidates.

Protein changes implicate the extended granin family, cell adhesion and insulin regulation in PD. An additional strength of DIA-MS analysis, and alternative quantitative proteomics strategies, is the ability to identify protein changes that may provide insight into PD disease mechanisms. The limited number of quantifiable proteins in the CSF (341 protein groups, Fig. 1) prompted a combined pathway analysis with significant proteins identified from both Cohort 1 and $2(\mathrm{p}<0.05 ; 95$ total) pooled together. The gene ontology bioprocess classification distribution was similar to the protein library for this subset of proteins (Additional File 1: Fig. S5a,b $)^{20,21}$. Using the CSF protein library (Fig. 1d) as the background proteome, enrichment $(\mathrm{p}<0.08)$ was observed for three bioprocesses, brain development, adherens junction organization, and homophilic cell adhesion via plasma membrane adhesion molecules (Additional File 1: Fig. S5c) ${ }^{26,27}$.

A total of 13 proteins (Top13) were found to change with a nominal $p<0.05$ consistently in both cohorts (Fig. 3a,b, highlighted in green; Additional Files 2, 3 and 5), 8 of which overlap with the 14 proteins described above that demonstrated the best predictive value in univariate analysis based on absolute odds ratio and AUC. Cohort 1 had a greater number of participants with cognitive impairment (MMSE $<22$ : Cohort $1=7$; Cohort $2=0$ ) and a higher average disease duration than Cohort 2 (Table 1), which might contribute to the small number of overlapping significant protein changes between cohorts. The age discrepancy between PD and HC in Cohort 2 may also contribute to the small number of overlapping protein changes observed in both cohorts. Three protein levels (APOD, antithrombin-III (SERPINC1), and C1QC) increased in PD CSF compared to HC; and the remaining 10 decreased (Fig. 3c,d). Significant elevation of SERPINC1 in CSF of PD patients was confirmed by ELISA analysis of both cohorts with a fold change of 1.3 and 1.4 for Cohort 1 and 2 , respectively ( $\mathrm{p}<0.05$, Additional File 1: Fig. S6).

The most enriched bioprocess in the Top13 is cell adhesion, including 3 proteins, $\mathrm{CDH} 2$, cell growth regulator with EF hand domain protein 1 (CGREF1), and ephrin type-A receptor 4 (EPHA4). CDH2 is directly involved in cell to cell adhesion by integrating into cellular membranes and forming a "reverse zipper" with other $\mathrm{CDH} 2$ proteins on adjacent cells ${ }^{28}$. CDH2 requires removal of its propeptide domain (aa 26-159) to be functionally active as a cell adhesion molecule ${ }^{29}$. Based on DIA-MS analysis, 7 peptides were identified in the small propeptide domain and zero peptides in the remainder of the protein which is over 700 amino acids long with over 50 basic 


\section{A Large-scale mass spectrometry (MS)-based biomarker discovery}

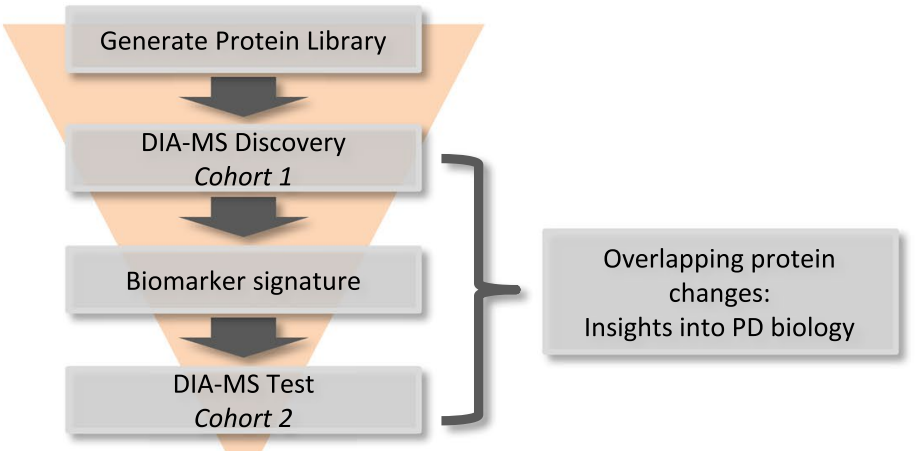

B Library Generation

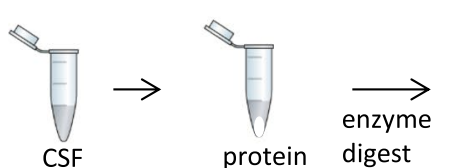

(PD, $n=4 ; H C, n=4)$ precipitation

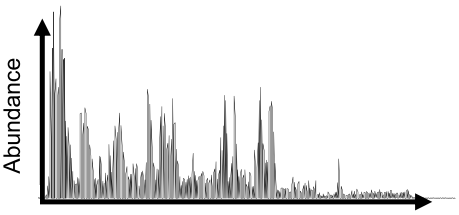

Retention Time DDA acquisition (60 min LC gradient)

C

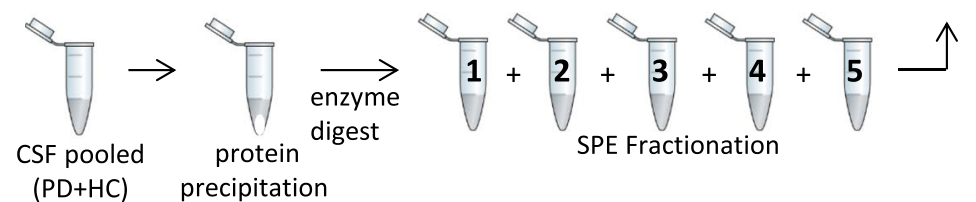

D CSF Protein Library

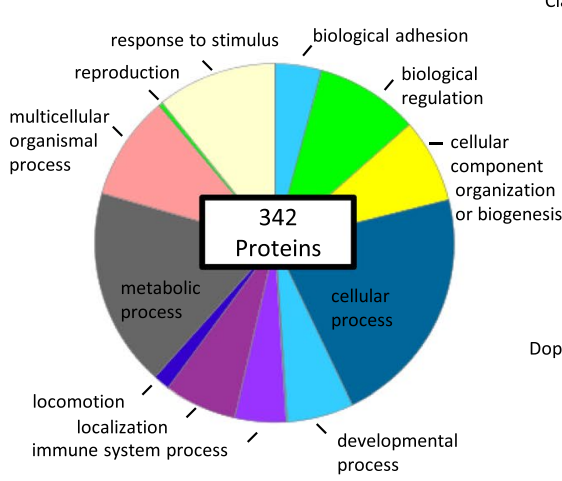

Clathrin-mediated Endocytosis

Sample Analysis

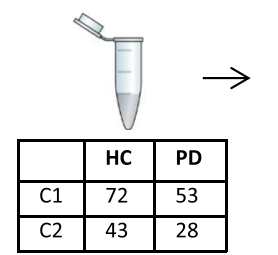

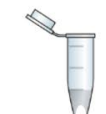

protein precipitation digest

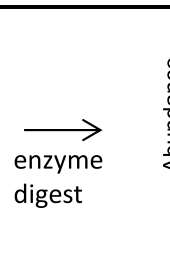

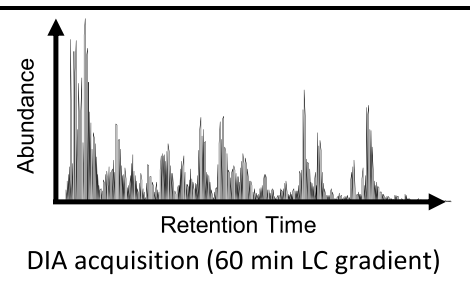

Figure 1. Large-scale mass spectrometry-based biomarker discovery. (a) DIA-MS approach overview. (b-e) Library generation and data acquisition workflow. To generate the library for processing samples by DIA-MS, a representative sample subset (b) and fractionated CSF pool (c) were processed and run in DDA-mode. (d) The gene ontology (go)-term bioprocess (left) classification (pantherdb.org) ${ }^{20,21}$ of the protein library consisting of 341 quantifiable protein groups. Proteins involved in pathways implicated in PD are indicated on the right. (e) CSF samples (see Table 1) were processed and analyzed by DIA-MS. A representative total ion chromatogram is shown on right. $\mathrm{SPE}=$ solid phase extraction, $\mathrm{DDA}=$ data-dependent acquisition.

residues available for enzymatic cleavage (Additional File 1: Fig. S7a). In agreement with these data, quantification by ELISA of the mature CDH2 (aa 160-906), which lacks the propeptide region, failed to detect any protein in CSF (Additional File 1: Fig. S7b,c). These data suggest the cleaved propeptide region is the only portion of 


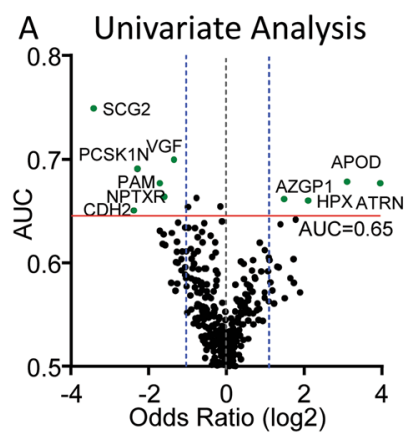

C
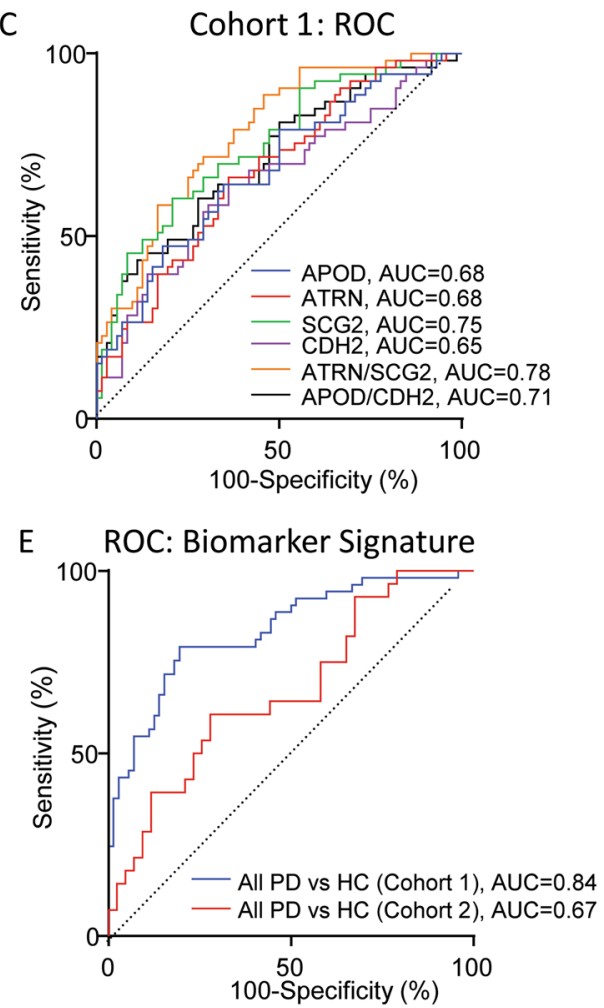

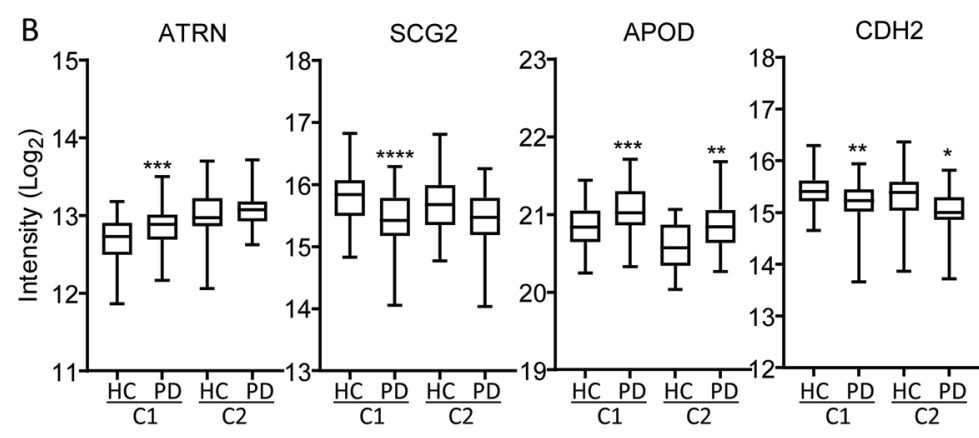

D

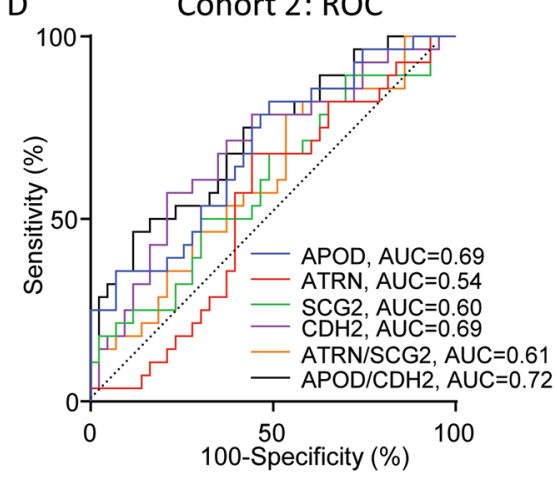

F Longitudinal Cohort

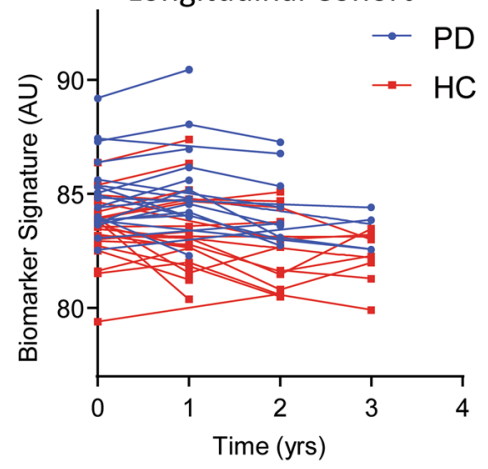

Figure 2. Univariate and multivariate analyses identify a PD-specific biomarker signature. (a) The univariate analyses of Cohort 1. Proteins highlighted in green and labeled by gene name have an AUC $>0.65$ (red line) and an odds ratio (OR) $>2$ or $<0.5$ (blue lines). (b) Whisker plots of the protein abundance of the top 4 predictive proteins. Significant protein changes of PD relative to the HC group are shown (Cohort $1=\mathrm{C} 1$; Cohort 2=C2). (c,d) ROC analysis of the top proteins and protein ratios identified by Cohort 1. (e) ROC analysis of the predictive biomarker signature, $(2.7(\mathrm{ATRN})+1.2(\mathrm{C} 1 \mathrm{QC})+1.7(\mathrm{APOD})-2.2(\mathrm{SCG} 2)+1.5(\mathrm{FBLN} 1))$.

(f) The calculated value of the biomarker signature as applied to the longitudinal cohort. $(* \mathrm{p}<0.05 ; * * \mathrm{p}<0.01$; $* * * \mathrm{p}<0.001 ; * * * \mathrm{p}<0.0001)$.

$\mathrm{CDH} 2$ present in CSF. The propeptide of $\mathrm{CDH} 2$ was decreased in $\mathrm{PD}$ compared to HC in both cohorts, suggesting a reduction in $\mathrm{CDH} 2$ processing, and therefore a decreased level of functionally active $\mathrm{CDH} 2$ in $\mathrm{PD}$. In addition, several studies have failed to identify changes in total $\mathrm{CDH} 2$ protein in post-mortem brain tissue, consistent with results in this study which suggest that a processing deficit, rather than change in total protein level, is altered in $\mathrm{PD}^{30,31}$.

Another Top13 protein found to decrease in PD relative to HC, receptor-type tyrosine-protein phosphatase N2 (PTPRN2), also known as phogrin and IA-2 $\beta$, is targeted to secretory granules ${ }^{32}$. PTPRN2 is a known regulator of insulin secretion ${ }^{33,34}$, and is implicated in type 1 diabetes $^{35}$. In the present study, PTPRN2 is decreased in PD relative to $\mathrm{HC}$, which may contribute to the link between insulin resistance and PD observed in certain patients ${ }^{36}$.

Three proteins found to decrease in PD relative to HC in both cohorts, neurosecretory protein VGF (VGF), secretogranin-3 (SCG3), and chromogranin-B (CHGB), are members of the extended granin family ${ }^{37}$. The granin family consists of 8 precursor proteins in total (VGF, SCG2, CHGB, SCG3, neuroendocrine protein 7B2 (SCG5), ProSAAS (PCSK1N), chromogranin-A (CHGA), neuroendocrine secretory protein 55 (GNAS)), all of which are known to be cleaved into functional peptides that undergo regulated secretion ${ }^{37,38}$. Additionally, CHGA is significantly reduced in Cohort $2(\mathrm{p}=0.040)$. Moreover, in Cohort 1 , which has almost double the number of samples 
A

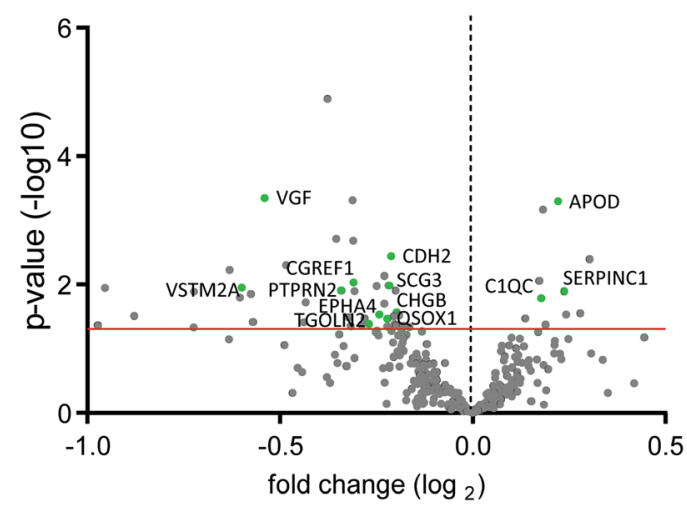

B

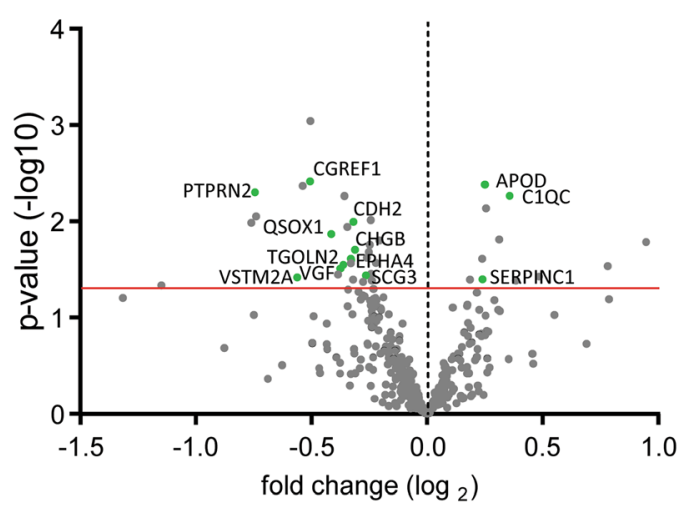

Cohort 2
C Proteins with significant changes

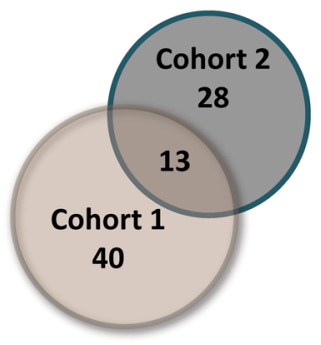

D

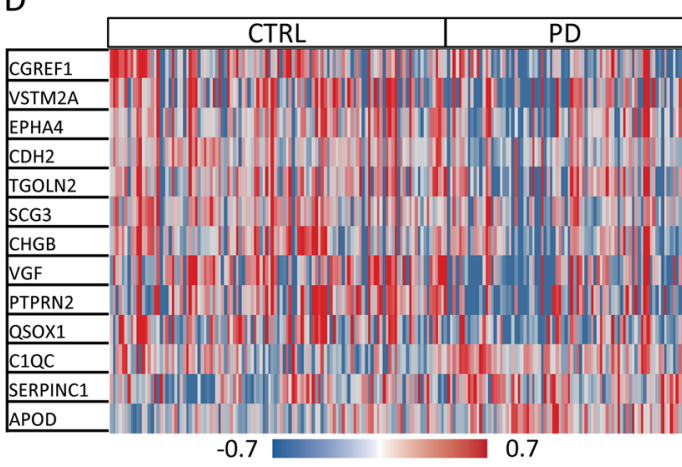

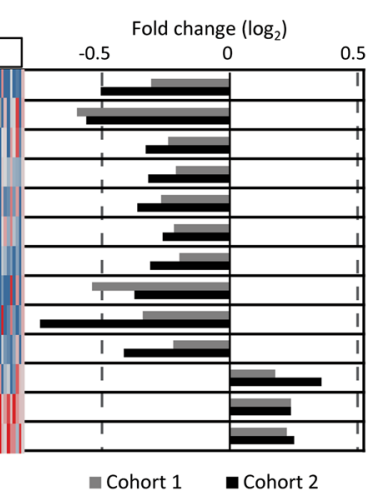

Figure 3. Thirteen proteins significantly change in both Cohort 1 and Cohort 2 in PD relative to HC. (a,b) Volcano plots of all proteins analyzed by DIA-MS for Cohort 1 (a) and Cohort 2 (b). Significant proteins identified in both cohorts are highlighted in green and labeled by gene name. The red line indicates a p-value cutoff of 0.05. (c) A Venn diagram displaying number of proteins with significant changes in Cohort 1 and 2. (d) Average fold change (right) compared to $\mathrm{HC}$ or individual sample (left) fold change normalized to mean for the 13 proteins (Top13) that were found to change in both cohorts by DIA-MS analysis in PD.

than Cohort 2, three additional granin family members, SCG2, PCSK1N, and SCG5, are reduced in PD relative to HC. The remaining family member, GNAS, was not detectable in CSF in our study (Additional Files 2 and 3 ).

The granin family members are known to be cleaved into bioactive peptides. To identify any region-specific and/or alterations in known bioactive peptides derived from the granin family members, we performed peptide level quantification of the 7 out of 8 granins that were detectable in the CSF. Only peptides that passed the manual inspection of spectra with an average $\mathrm{q}<0.005$ were considered in the analysis. All 7 proteins had peptides that were significantly decreased in PD relative to $\mathrm{HC}$ in at least one cohort (Fig. 4, Additional File 6). SCG2, a granin family member found to significantly decrease only in Cohort 1 at the protein level, has two C-terminal region peptides that are significantly decreased in both cohorts in the peptide level analysis. This region corresponds to the bioactive peptide manserin, which is known to be present in the neuroendocrine system and cerebellum. Additionally, several peptides do not change between PD and HC in either cohort suggesting that the total protein level of this extended granin family is unchanged, but processing, localization and/or secretion of these peptides is likely affected. Although further investigation is required to confirm this hypothesis, there is additional evidence for altered peptide processing in PD (see discussion).

\section{Discussion}

The present study employed DIA-MS proteomics to identify protein changes in PD CSF. This quantitative approach allowed the identification of unique alterations of several biomarker candidates (as protein ratios or detection signatures). Analyses of significant protein changes implicated the granin family, peptide processing, insulin regulation and cell adhesion in PD.

The findings presented in this study suggest an altered granin family metabolism in PD. Numerous studies have implicated a subset of peptides derived from the granin family in neurodegenerative diseases ${ }^{33,39-45}$, the present study expands on these observations, associating nearly the entire extended granin family with PD. We observed the reduction of multiple granins that function as biologically active peptides in PD relative to HC (i.e., VGF, CHGA, CHGB, SCG2, SCG3, SCG5, and PCSK1N). The granin family plays an essential role in the regulated secretory pathway that is responsible for controlled delivery of peptides and neurotransmitters ${ }^{37}$. Importantly, granin peptides and catecholamines are co-stored in dense-core vesicles and granins can regulate levels and functions of catecholamines ${ }^{46-48}$, such as dopamine which is reduced in PD. The reduction of 


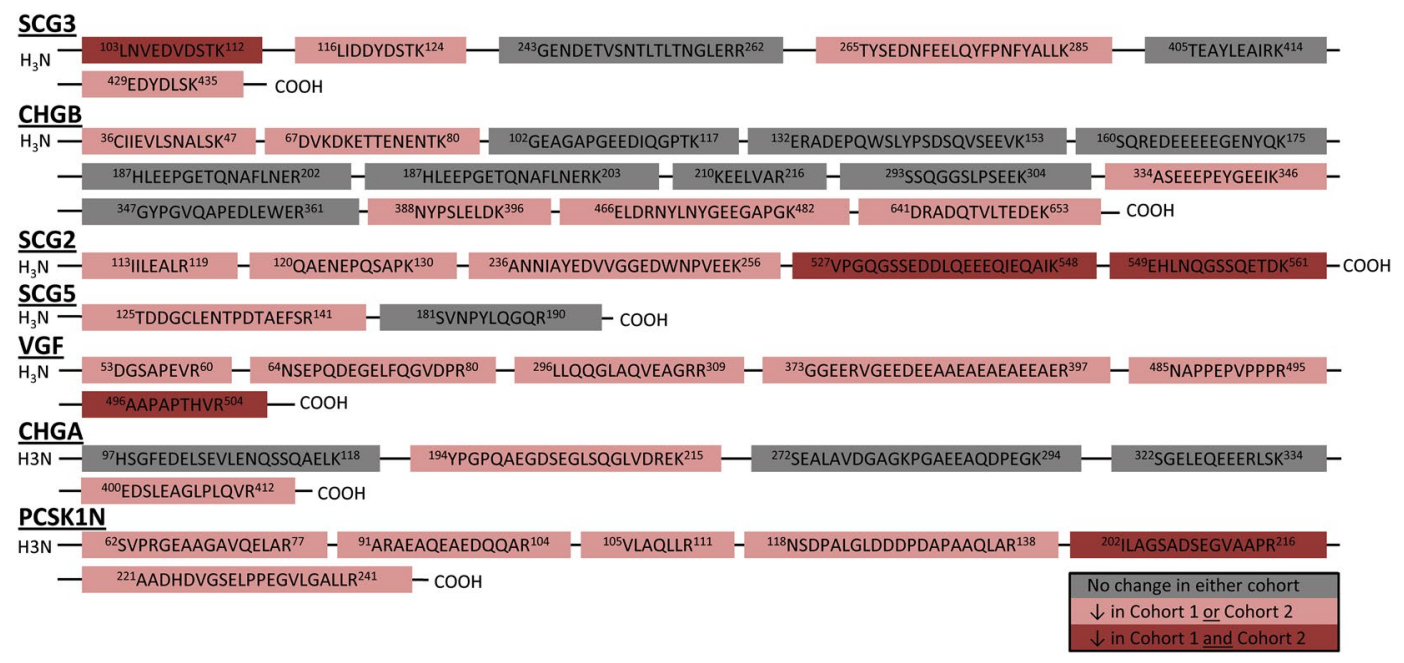

Figure 4. Peptide quantification reveals region-specific reduction of the extended granin family in PD. Granin peptides were found to either decrease in PD in both cohorts (red), one cohort (light red), or neither cohort (gray). The C-terminal region of PCSK1N, SCG2, and VGF are decreased in both PD cohorts relative to HC. For SCG3, the N-terminal region is decreased in PD.

granin-derived biologically active peptides observed in this study might have pathophysiological implications and supports the larger catecholaminergic deficit in PD. Correspondingly, alterations in granin levels or metabolism have been reported in neurotoxin-induced models of $\mathrm{PD}^{42-44}$.

Alterations in specific aspects of granin biology have been reported in the context of multiple neurodegenerative diseases. Region-specific mislocalization of peptide fragments of the granin family of proteins to pathological inclusions have been observed in brain tissue from pathologically confirmed AD, PD, and Pick's disease ${ }^{39,41,49}$. Deposition of alpha-synuclein, the pathological hallmark of PD, was recently found on secretogranin II-positive vesicular membranes, suggesting a mechanistic link between the reduction of granins in CSF and PD pathogene$\mathrm{sis}^{50}$. Previous studies have also reported distinct metabolic processing of specific granins in CSF. The N-terminal region of VGF was decreased in frontotemporal dementia whereas its C-terminal region is reduced in $\mathrm{AD}^{40,45}$. Interestingly, the C-terminal region of VGF was decreased in the CSF of PD patients in both cohorts in this study.

Further mechanistic support to the extensive misprocessing of granin peptides is provided by the reduction in peptidyl-glycine alpha-amidating monooxygenase (PAM). Activation of approximately half of bioactive peptides, including the granins, is mediated by PAM, a C-terminal alpha-amidating enzyme. PAM levels were reduced in $\mathrm{PD}$ relative to $\mathrm{HC}$ in Cohort $1(\mathrm{p}=0.002)$ and in the less severely affected Cohort $2(\mathrm{p}=0.066)$, further implicating altered precursor processing in PD. PAM is located in secretory granules with VGF, which contains a canonical PAM C-terminal alpha-amidation site. A bioactive peptide of CHGA (namely PST) requires PAM-mediated $\mathrm{C}$-terminal amidation for activation ${ }^{37}$. The decrease in the propeptide region of $\mathrm{CDH} 2$ in the present study, further implicates altered peptide processing in the context of PD. Lack of $\mathrm{CDH} 2$ processing is known to reduce cell-to-cell adhesion efficiency ${ }^{28,51}$ which might in turn contribute to increased blood-brain barrier permeability.

The protein changes identified in this study provide additional support to the impaired insulin regulation in $\mathrm{PD}$, including in PD dementia ${ }^{36}$. "Anti-insulin resistance" therapies are under investigation as therapeutic strate-

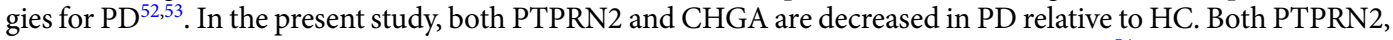
as well as the CHGA derived peptide, PST, are known modulators of insulin secretion ${ }^{54}$, providing a potential mechanistic explanation to the insulin resistance in $\mathrm{PD}^{36,52,53}$. Evaluation of larger clinically defined cohorts would be required to define the association of particular protein aberrations with specific disease phenotypes.

Bioprocess enrichment analysis identified adherens junction organization as the most over-represented biological process, representing a total of 6 proteins that were decreased in PD relative to HC: cadherins $2,6,8$, 10 and 13 (CDH2, CDH6, CDH 8, CDH10 and CDH 13), and cell adhesion molecule 2 (CADM2). Adherens junction, along with gap and tight junctions, play a key role in the maintenance and permeability of the blood brain barrier ${ }^{55}$. In accord with the present study, system biology approaches have also identified alterations in the adherens junction in $\mathrm{PD}^{56-58}$, which might be related to the blood brain barrier compromise in $\mathrm{PD}^{59,60}$.

There are several limitations associated with this study that require further exploration. First, over $90 \%$ of $\mathrm{PD}$ patients were prescribed levodopa and/or a dopamine agonist and their contribution to the protein changes described herein has not been assessed, requiring a de novo PD sample set for evaluation. Second, although the standardization in DIA-MS provides acceptable peptide quantification for the peptides present in the protein library, it is quite possible that enhancing the protein library with in-depth fractionation of CSF may translate to more quantifiable proteins in the analysis of the CSF sample cohorts. Quantifying the protein changes identified by an orthogonal method would be required to confirm the findings identified in this study as well. We were able to confirm SERPINC1 elevation in PD CSF by ELISA, but were unable to extend this analysis to the granin family due to limited availability of samples and suitable antibodies specific to the identified peptides. Lastly, an independent CSF cohort would be required to determine the robustness of the protein changes observed in the longitudinal cohort. 


\section{Conclusion}

The present study identifies potential PD biomarkers and novel protein changes implicating cell adhesion, insulin regulation, and bioactive peptide processing. Importantly, the extended granin family was reduced in disease, suggesting a potential common mechanism for the biological reduction in monoamine neurotransmission in Parkinson's patients. With several promising disease-modifying therapeutics under development ${ }^{3,4}$, there is an increased need for objective biomarkers to evaluate their efficacy. The continuous development of robust platforms to quantify small changes in proteins in biospecimens as well as the evaluation of larger longitudinal cohorts are critical for future patient subtyping and evaluation of therapeutics.

\section{Data availability}

All output files (.raw), search file (.sne), Swiss-prot export used in library and sample processing (.fasta), spectral library (.kit), and protein quantification data (.xlsx) have been deposited to PRIDE under the accession number: PXD011216.

Received: 27 September 2019; Accepted: 24 January 2020;

Published online: 12 February 2020

\section{References}

1. Titova, N. \& Chaudhuri, K. R. Non-motor Parkinson disease: new concepts and personalised management. Med. J. Aust. 208, 404-409 (2018).

2. Poewe, W. et al. Parkinson disease. Nat. Rev. Dis. Prim. 3, 17013, https://doi.org/10.1038/nrdp.2017.13 (2017).

3. Lang, A. E. \& Espay, A. J. Disease Modification in Parkinson's Disease: Current Approaches, Challenges, and Future Considerations. Mov. Disord. 33, 660-677, https://doi.org/10.1002/mds.27360 (2018).

4. Sardi, S. P., Cedarbaum, J. M. \& Brundin, P. Targeted Therapies for Parkinson's Disease: From Genetics to the Clinic. Mov. Disord. 33, 684-696, https://doi.org/10.1002/mds.27414 (2018).

5. Olsson, B. et al. CSF and blood biomarkers for the diagnosis of Alzheimer's disease: a systematic review and meta-analysis. Lancet Neurol. 15, 673-684, https://doi.org/10.1016/S1474-4422(16)00070-3 (2016).

6. Blennow, K. et al. Clinical utility of cerebrospinal fluid biomarkers in the diagnosis of early Alzheimer's disease. Alzheimers Dement. 11, 58-69, https://doi.org/10.1016/j.jalz.2014.02.004 (2015).

7. Hu, A., Noble, W. S. \& Wolf-Yadlin, A. Technical advances in proteomics: new developments in data-independent acquisition. F1000Res 5, https://doi.org/10.12688/f1000research.7042.1 (2016).

8. Dawani, K., Tayyab, M. \& Karira, K. A. Serum and CSF immunoglobulins G, A and M in 37 intracranial tumors. J. Pak. Med. Assoc. 52, 549-553 (2002).

9. Pisani, V. et al. Increased blood-cerebrospinal fluid transfer of albumin in advanced Parkinson's disease. J. Neuroinflammation 9, 188, https://doi.org/10.1186/1742-2094-9-188 (2012).

10. Sinha, A. et al. Identification of differentially displayed proteins in cerebrospinal fluid of Parkinson's disease patients: a proteomic approach. Clin. Chim. Acta 400, 14-20, https://doi.org/10.1016/j.cca.2008.09.026 (2009).

11. Guo, J. et al. Proteomic analysis of the cerebrospinal fluid of Parkinson's disease patients. Cell Res. 19, 1401-1403, https://doi. org/10.1038/cr.2009.131 (2009).

12. Abdi, F. et al. Detection of biomarkers with a multiplex quantitative proteomic platform in cerebrospinal fluid of patients with neurodegenerative disorders. J. Alzheimers Dis. 9, 293-348 (2006).

13. Callister, S. J. et al. Normalization approaches for removing systematic biases associated with mass spectrometry and label-free proteomics. J. Proteome Res. 5, 277-286, https://doi.org/10.1021/pr0503001 (2006).

14. Mohammadi, D. The Harvard Biomarker Study's big plan. Lancet Neurol. 12, 739-740, https://doi.org/10.1016/S14744422(13)70155-8 (2013).

15. Liu, G. et al. Prediction of cognition in Parkinson's disease with a clinical-genetic score: a longitudinal analysis of nine cohorts. Lancet Neurol. 16, 620-629, https://doi.org/10.1016/S1474-4422(17)30122-9 (2017).

16. Liu, G. et al. Specifically neuropathic Gaucher's mutations accelerate cognitive decline in Parkinson's. Ann. Neurol. 80, 674-685, https://doi.org/10.1002/ana.24781 (2016).

17. Kang, U. J. et al. The BioFIND study: Characteristics of a clinically typical Parkinson's disease biomarker cohort. Mov. Disord. 31, 924-932, https://doi.org/10.1002/mds.26613 (2016).

18. Benjamini, Y. \& Hochberg, Y. Controlling the false discovery rate: a practical and powerful approach to multiple testing. J. R. Stat. Soc. 57, 289-300 (1995).

19. Tibshirani, R. Regression Shrinkage and Selection via the Lasso. J. R. Stat. Soc. 58, 267-288 (1996).

20. Mi, H. \& Thomas, P. PANTHER pathway: an ontology-based pathway database coupled with data analysis tools. Methods Mol. Biol. 563, 123-140, https://doi.org/10.1007/978-1-60761-175-2_7 (2009).

21. Mi, H., Muruganujan, A., Ebert, D., Huang, X. \& Thomas, P. D. PANTHER version 14: more genomes, a new PANTHER GO-slim and improvements in enrichment analysis tools. Nucleic Acids Res. 47, D419-D426, https://doi.org/10.1093/nar/gky1038 (2019).

22. Hall, S. et al. CSF biomarkers and clinical progression of Parkinson disease. Neurol. 84, 57-63, https://doi.org/10.1212/ WNL.0000000000001098 (2015).

23. Maass, F., Schulz, I., Lingor, P., Mollenhauer, B. \& Bahr, M. Cerebrospinal fluid biomarker for Parkinson's disease: An overview. Mol. Cell Neurosci. 97, 60-66, https://doi.org/10.1016/j.mcn.2018.12.005 (2019).

24. Street, J. M. et al. Identification and proteomic profiling of exosomes in human cerebrospinal fluid. J. Transl. Med. 10, 5, https://doi. org/10.1186/1479-5876-10-5 (2012).

25. Waldner, A. et al. Concentration in Human Plasma during Aging and in Parkinson's Disease: A Cross-Sectional Study. Parkinsons Dis. 2018, 3751516, https://doi.org/10.1155/2018/3751516 (2018).

26. Huang da, W., Sherman, B. T. \& Lempicki, R. A. Systematic and integrative analysis of large gene lists using DAVID bioinformatics resources. Nat. Protoc. 4, 44-57, https://doi.org/10.1038/nprot.2008.211 (2009).

27. Huang da, W., Sherman, B. T. \& Lempicki, R. A. Bioinformatics enrichment tools: paths toward the comprehensive functional analysis of large gene lists. Nucleic Acids Res. 37, 1-13, https://doi.org/10.1093/nar/gkn923 (2009).

28. Gumbiner, B. M. Regulation of cadherin-mediated adhesion in morphogenesis. Nat. Rev. Mol. Cell Biol. 6, 622-634, https://doi. org/10.1038/nrm1699 (2005).

29. Maret, D. et al. Opposite roles of furin and PC5A in N-cadherin processing. Neoplasia 14, 880-892 (2012).

30. Shi, M. et al. Identification of glutathione S-transferase pi as a protein involved in Parkinson disease progression. Am. J. Pathol. 175, 54-65, https://doi.org/10.2353/ajpath.2009.081019 (2009).

31. Shi, M. et al. Mortalin: a protein associated with progression of Parkinson disease? J. Neuropathol. Exp. Neurol. 67, 117-124, https:// doi.org/10.1097/nen.0b013e318163354a (2008). 
32. Wasmeier, C., Bright, N. A. \& Hutton, J. C. The lumenal domain of the integral membrane protein phogrin mediates targeting to secretory granules. Traffic 3, 654-665 (2002).

33. Kubosaki, A. et al. Targeted disruption of the IA-2beta gene causes glucose intolerance and impairs insulin secretion but does not prevent the development of diabetes in NOD mice. Diabetes 53, 1684-1691 (2004).

34. Caromile, L. A., Oganesian, A., Coats, S. A., Seifert, R. A. \& Bowen-Pope, D. F. The neurosecretory vesicle protein phogrin functions as a phosphatidylinositol phosphatase to regulate insulin secretion. J. Biol. Chem. 285, 10487-10496, https://doi.org/10.1074/jbc. M109.066563 (2010).

35. $\mathrm{Lu}$, J. et al. Identification of a second transmembrane protein tyrosine phosphatase, IA-2beta, as an autoantigen in insulin-dependent diabetes mellitus: precursor of the 37-kDa tryptic fragment. Proc. Natl Acad. Sci. USA 93, 2307-2311 (1996).

36. Bosco, D. et al. Dementia is associated with insulin resistance in patients with Parkinson's disease. J. Neurol. Sci. 315, 39-43, https:// doi.org/10.1016/j.jns.2011.12.008 (2012)

37. Bartolomucci, A. et al. The extended granin family: structure, function, and biomedical implications. Endocr. Rev. 32, 755-797, https://doi.org/10.1210/er.2010-0027 (2011).

38. Taupenot, L., Harper, K. L. \& O’Connor, D. T. The chromogranin-secretogranin family. N. Engl. J. Med. 348, 1134-1149, https://doi. org/10.1056/NEJMra021405 (2003)

39. Wada, M. et al. A human granin-like neuroendocrine peptide precursor (proSAAS) immunoreactivity in tau inclusions of Alzheimer's disease and parkinsonism-dementia complex on Guam. Neurosci. Lett. 356, 49-52 (2004).

40. Carrette, O. et al. A panel of cerebrospinal fluid potential biomarkers for the diagnosis of Alzheimer's disease. Proteom. 3, 1486-1494, https://doi.org/10.1002/pmic.200300470 (2003)

41. Cocco, C. et al. Distribution of VGF peptides in the human cortex and their selective changes in Parkinson's and Alzheimer's diseases. J. Anat. 217, 683-693, https://doi.org/10.1111/j.1469-7580.2010.01309.x (2010).

42. Li, F. et al. Dysregulated expression of secretogranin III is involved in neurotoxin-induced dopaminergic neuron apoptosis. J. Neurosci. Res. 90, 2237-2246, https://doi.org/10.1002/jnr.23121 (2012).

43. Medhurst, A. D. et al. Up-regulation of secretoneurin immunoreactivity and secretogranin II mRNA in rat striatum following 6-hydroxydopamine lesioning and chronic L-DOPA treatment. Neurosci. 105, 353-364 (2001).

44. Nilsson, A. et al. Striatal alterations of secretogranin-1, somatostatin, prodynorphin, and cholecystokinin peptides in an experimental mouse model of Parkinson disease. Mol. Cell Proteom. 8, 1094-1104, https://doi.org/10.1074/mcp.M800454-MCP200 (2009).

45. Ruetschi, U. et al. Identification of CSF biomarkers for frontotemporal dementia using SELDI-TOF. Exp. Neurol. 196, 273-281, https://doi.org/10.1016/j.expneurol.2005.08.002 (2005).

46. Diaz-Vera, J. et al. Chromogranins A and B are key proteins in amine accumulation, but the catecholamine secretory pathway is conserved without them. FASEB J. 26, 430-438, https://doi.org/10.1096/f.11-181941 (2012).

47. Diaz-Vera, J. et al. Chromogranin B gene ablation reduces the catecholamine cargo and decelerates exocytosis in chromaffin secretory vesicles. J. Neurosci. 30, 950-957, https://doi.org/10.1523/JNEUROSCI.2894-09.2010 (2010).

48. Montesinos, M. S. et al. The crucial role of chromogranins in storage and exocytosis revealed using chromaffin cells from chromogranin A null mouse. J. Neurosci. 28, 3350-3358, https://doi.org/10.1523/JNEUROSCI.5292-07.2008 (2008).

49. Kikuchi, K. et al. An N-terminal fragment of ProSAAS (a granin-like neuroendocrine peptide precursor) is associated with tau inclusions in Pick's disease. Biochem. Biophys. Res. Commun. 308, 646-654 (2003).

50. Brekk, O. R., Moskites, A., Isacson, O. \& Hallett, P. J. Lipid-dependent deposition of alpha-synuclein and Tau on neuronal Secretogranin II-positive vesicular membranes with age. Sci. Rep. 8, 15207, https://doi.org/10.1038/s41598-018-33474-z (2018).

51. Maret, D. et al. Surface expression of precursor N-cadherin promotes tumor cell invasion. Neoplasia 12, 1066-1080 (2010).

52. Aviles-Olmos, I., Limousin, P., Lees, A. \& Foltynie, T. Parkinson's disease, insulin resistance and novel agents of neuroprotection. Brain 136, 374-384, https://doi.org/10.1093/brain/aws009 (2013).

53. Takamatsu, Y. et al. Combined immunotherapy with "anti-insulin resistance" therapy as a novel therapeutic strategy against neurodegenerative diseases. NPJ Parkinsons Dis. 3, 4, https://doi.org/10.1038/s41531-016-0001-1 (2017).

54. Tatemoto, K. et al. Pancreastatin, a novel pancreatic peptide that inhibits insulin secretion. Nat. 324, 476-478, https://doi. org/10.1038/324476a0 (1986).

55. Stamatovic, S. M., Johnson, A. M., Keep, R. F. \& Andjelkovic, A. V. Junctional proteins of the blood-brain barrier: New insights into function and dysfunction. Tissue Barriers 4, e1154641, https://doi.org/10.1080/21688370.2016.1154641 (2016).

56. Edwards, Y. J. et al. Identifying consensus disease pathways in Parkinson's disease using an integrative systems biology approach. PLoS One 6, e16917, https://doi.org/10.1371/journal.pone.0016917 (2011).

57. Chandrasekaran, S. \& Bonchev, D. A network view on Parkinson's disease. Comput. Struct. Biotechnol. J. 7, e201304004, https://doi. org/10.5936/csbj.201304004 (2013).

58. Karim, S., Mirza, Z., Kamal, M. A., Abuzenadah, A. M. \& Al-Qahtani, M. H. Gene expression analysis approach to establish possible links between Parkinson's disease, cancer and cardiovascular diseases. CNS Neurol. Disord. Drug. Targets 13, 1334-1345, https://doi. org/10.2174/1871527313666141023122803 (2014)

59. Desai, B. S., Monahan, A. J., Carvey, P. M. \& Hendey, B. Blood-brain barrier pathology in Alzheimer's and Parkinson's disease: implications for drug therapy. Cell Transpl. 16, 285-299, https://doi.org/10.3727/000000007783464731 (2007).

60. Sweeney, M. D., Sagare, A. P. \& Zlokovic, B. V. Blood-brain barrier breakdown in Alzheimer disease and other neurodegenerative disorders. Nat. Rev. Neurol. 14, 133-150, https://doi.org/10.1038/nrneurol.2017.188 (2018).

\section{Acknowledgements}

We thank all study participants, their families, and friends for their support and participation, and our study coordinators. C.R.S.'s work was supported in part by NIH grants U01NS082157, U01NS095736, U01NS100603, R01AG057331 and the American Parkinson's Disease Association. HBS was supported in part by the Harvard NeuroDiscovery Center, MJFF, and the Massachusetts Alzheimer's Disease Research Center NIA P50AG005134. For up-to-date information on the study, visit https://www.bwhparkinsoncenter.org/biobank/. The CSF collection at CUMC was supported by MJFF BioFind study. Biospecimen and data used in preparation of this article were obtained from the Fox Investigation for New Discovery of Biomarkers ("BioFIND”), but were not stored at the BioFIND centralized biorepository and database. BioFIND is sponsored by The Michael J. Fox Foundation for Parkinson's Research (MJFF) with support from the National Institute for Neurological Disorders and Stroke (NINDS). For up-to-date information on the study, visit www.michaeljfox.org/biofind. We thank the following Studies and Investigators: Harvard Biomarkers Study. Co-Directors: Brigham and Women's Hospital: Clemens R. Scherzer, Charles Jennings; Massachusetts General Hospital: Bradley T. Hyman; Investigators and Study Coordinators: Brigham and Women's Hospital: Yuliya Kuras, Daly Franco; Brigham and Women's Hospital: Michael T. Hayes, Chizoba C. Umeh, Vikram Khurana, Reisa Sperling; Massachusetts General Hospital: John H. Growdon, Michael A. Schwarzschild, Albert Y. Hung, Alice W. Flaherty, Deborah Blacker, Anne-Marie Wills, 
U. Shivraj Sohur, Vivek K. Unni, Nicte I. Mejia, Anand Viswanathan, Stephen N. Gomperts, Mark W. Albers, Maria Allora-Palli, Alireza Atri, David Hsu, Alexandra Kimball, Scott McGinnis, Nutan Sharma, John Becker, Randy Buckner, Thomas Byrne, Maura Copeland, Bradford Dickerson, Matthew Frosch, Theresa Gomez-Isla, Steven Greenberg, James Gusella, Julius Hedden, Elizabeth Hedley-Whyte, Keith Johnson, Raymond Kelleher, Aaron Koenig, Maria Marquis-Sayagues, Gad Marshall, Sergi Martinez-Ramirez, Donald McLaren, Olivia Okereke, Elena Ratti, Christopher William, Koene Van Dij, Shuko Takeda, Anat Stemmer-Rachaminov, Jessica Kloppenburg, Catherine Munro, Rachel Schmid, Sarah Wigman, Sara Wlodarcsyk; Scientific Advisory Board: Massachusetts General Hospital: John H. Growdon; Brigham and Women's Hospital: Dennis J. Selkoe, Reisa Sperling; Data Coordination: Brigham and Women's Hospital: Thomas Yi, Joseph J. Locascio; Massachusetts General Hospital: Haining Li; Biobank Management Staff: Brigham and Women's Hospital: Gabriel Stalberg, Zhixiang Liao. M.S.R., M.L., W.Z., P.W., P.O., C.V., L.S., K.Z. and SPS are/were employees of Sanofi. C.R.S. has collaborated with Pfizer, Proteome Sciences, Sanofi; has consulted for Sanofi; has served as Advisor to the Michael J. Fox Foundation, NIH, Department of Defense; is on the Scientific Advisory Board of the American Parkinson Disease Association; has received funding from the NIH, the U.S. Department of Defense, the Michael J. Fox Foundation, American Parkinson Disease Association. C.R.S. and P.S. are named as co-inventors on a US patent application on sphingolipids biomarkers for proteinopathies that is jointly held by Brigham \& Women's Hospital and Sanofi. A.M.W. has received research funding from the A.L.S. Association, has participated in clinical trials funded by Acorda, Biogen, Bristol-Myers Squibb, Sanofi/Genzyme, Pfizer and received consultant payments from Accordant, a CVS/Caremark disease management company. R.N.A. is supported by the Parkinson's Foundation, the National Institutes of Health and the Michael J Fox Foundation. RNA consulted to Genzyme/Sanofi, Biogen, Denali and Prophase.

\section{Author contributions}

(1) Research Project: A. Conception and Design; B. Acquisition of Data; C. Analysis and Interpretation of Data; (2) Manuscript: A. Writing of the First Draft, B. Review and Critique; (3) Other: A. Statistical Analysis; B. Obtaining Funding; C. Technical Support; D. Supervision of Data Collection. MR: 1A, 1B, 1C, 2A, 2B, 3A. WZ: 3A. ML: 1A, 3C. PW: 1A, 3C. PO: 3D. CV: 3C. AW: 3C. CS: 2B. RA: 2B. LS: 2B. KZ: 1A, 2B. PS: 1A, 2B, 3B. All authors read and approved the final manuscript

\section{Competing interests}

The authors declare no competing interests.

\section{Additional information}

Supplementary information is available for this paper at https://doi.org/10.1038/s41598-020-59414-4.

Correspondence and requests for materials should be addressed to S.P.S.

Reprints and permissions information is available at www.nature.com/reprints.

Publisher's note Springer Nature remains neutral with regard to jurisdictional claims in published maps and institutional affiliations.

(c) (i) Open Access This article is licensed under a Creative Commons Attribution 4.0 International License, which permits use, sharing, adaptation, distribution and reproduction in any medium or format, as long as you give appropriate credit to the original author(s) and the source, provide a link to the Creative Commons license, and indicate if changes were made. The images or other third party material in this article are included in the article's Creative Commons license, unless indicated otherwise in a credit line to the material. If material is not included in the article's Creative Commons license and your intended use is not permitted by statutory regulation or exceeds the permitted use, you will need to obtain permission directly from the copyright holder. To view a copy of this license, visit http://creativecommons.org/licenses/by/4.0/.

(C) The Author(s) 2020 